\title{
Effects of chronic illness on children and their families
}

\section{Christine Eiser}

The proportion of children affected by chronic illness is relatively high, approximately $10-12 \%$. For these children, there is no available cure and the best that medical care can currently offer is relief from symptoms and the opportunity to participate in normal life as far as possible. Epidemiological work suggests that many of the children experience relatively few restrictions on everyday life, but about $1-2 \%$ of the total population of sick children have more severe conditions (Gortmaker \& Sappenfield, 1984).

Advances in biotechnology mean that it is now possible to treat many more children than in the past. However, the financial cost to the health service is coming under greater scrutiny, and the cost to families in social and psychological terms is increasingly recognised. Costs for families include the added care-taking burden involved in care of a sick child, increased psychological morbidity, especially among mothers, and impact on other family members, especially healthy siblings. For the child, there are likely to be interruptions to regular schooling, restricted mobility and activities, and sometimes long-term restrictions into adult life (Eiser, 1995). The need for regular medical treatment may well compromise normal childhood activities.

In the past, there has been a tendency for psychologists to work within a medical model in child health psychology. Thus, it is assumed that the consequences of chronic illness for psychological health are disease-specific. Such a view is perpetuated where psychologists are 'attached' to clinical specialities, for example in oncology or renal disease. Reservations about this approach have been expressed. A disease-specific model has a number of disadvantages (Box 1; see Stein et al,
1993). First, it is not possible to include every possible disorder to which children might be susceptible. Second, diagnoses may be made inconsistently by different physicians (this applies especially to conditions such as asthma). Third, a diagnostic label is relatively uninformative with regard to the severity of the condition. Fourth, there may be a bias to include only those children who have access to the medical care system; and fifth, there may be a delay between the emergence of symptoms and diagnosis. In many instances, it may therefore be more useful to categorise diseases in terms of their consequences for children rather than their labels.

Early work concerned with the psychosocial impact of chronic illness in children took what has been described as a 'deficit centred' approach (Drotar, 1978), that is it was assumed that the child would inevitably show emotional and behavioural deficits. It has subsequently been argued that such a simple cause-effect model is inadequate to describe the range of outcomes that have been identified. Current theoretical approaches emphasise the multi-dimensional ways in which children respond. Wallander \& Thompson (1995), for example, describe a set of 'resistance' and 'resilience' factors which buffer the child from maladjustment. Such models have been useful in promoting a wider and more balanced approach to considering psychosocial outcomes.

Even so, the literature is far from clear regarding the extent to which children show maladjustment. Conventional literature reviews and meta-analyses (Lavigne \& Faier-Routman, 1992) highlight the discrepancies in the literature. While some work supports the conventional notion that chronically sick children show emotional and behavioural

Christine Eiser is Reader in Health Psychology, CRC Child and Family Research Group, Department of Psychology, University of Exeter, Exeter, Devon EX4 4GQ. She has published on all aspects of the psychological impact of chronic diseases on children and their families. Currently, she is especially concerned to develop simple child-friendly measures which can be used in evaluations of clinical trials, interventions, etc. 
Box 1. Chronic diseases share the following characteristics

They have a biological, psychological or cognitive basis

They last, or are virtually certain to last, for more than one year

They are associated with one or more of the following sequelae:

(a) Limitation of function, activities or social role in comparison with healthy, same-age peers in the areas of physical, cognitive, emotional and social functioning, as well as growth and development

(b) Dependency on one or more of the following to compensate for or minimise limitation of function, activities or social role: medications, special diet, medical technology, assistive device, personal assistance

(c) A need for medical care or related services, psychological services or educational services over and above the usual for the child's age, or for special ongoing treatments, interventions or accommodations at home or school (see Stein et al, 1993, p. 345.)

problems, it is often difficult to distinguish sick children from normal samples, and occasionally it is reported that sick children perform better than normals. Attempts to reconcile these discrepant findings focus on methodological issues, especially differences in sampling and assessment.

The measures in common use are far from ideal and were often developed for different groups of children, including those with psychiatric diagnoses and frank psychopathology. They can be long and repetitive to use with children who are sick. They often lack sensitivity toward the particular issues of concern. Recognition of these limitations has led to attempts to develop measures based more directly on information provided by the children themselves. Simple quality of life measures that can be completed by children are in demand for use in evaluating interventions and as indicators of psychological morbidity associated with different treatment regimens or in clinical trials (Eiser \& Jenney, 1996). While progress has been made in developing instruments for use with adolescents, this is less true for younger children.

The appropriateness of the traditional model, in which sick children are compared with healthy controls on a number of dimensions, is increasingly being challenged. Of the control groups used (healthy peers, siblings, published norms) none is entirely satisfactory. This approach seems to suggest that the behaviour of healthy children is a 'gold standard' against which others can be measured.

There are many other issues of concern in addition to psychosocial function. Many of these are poorly studied, partly because there is no obvious standardised instrument available. Partly as a reaction against the scientific approach and partly because some of the most interesting and clinically relevant questions do not lend themselves to conventional methods, some workers are turning to more 'qualitative' approaches to eliciting data. While these undoubtedly offer much more detailed information about the impact of disease from the child's perspective, they are open to criticism regarding the replicability of the data.

\section{Communication}

The question of communication with the sick child is a case in point. Some years ago, when children with chronic conditions generally had very poor prognosis, it was argued that open discussion of the illness was inappropriate. Information was thought to be harmful to the child, creating unnecessary anxiety and often resulting in children showing difficult and non-compliant behaviour. Several factors forced a change in this approach. First, medical care improved to the extent that many children survived. This in itself may have meant that adults felt more comfortable about discussing the implications of illness with children. Second, in many chronic conditions part of the goal of treatment is to encourage children to become more responsible for the management of their own disease. Although conditions vary in the extent to which patients need to look after themselves, most paediatricians like to foster some individual responsibility. In some conditions, notably diabetes, good health is considered largely dependent on self-care. Issues of communication with the child become paramount.

\section{At diagnosis}

Many advocate that children should be told about their disease, arguing that information reduces 
anxiety and fear, and promotes compliant and adaptive behaviour Despite the widespread adoption of this view, the empirical evidence that 'telling' is associated with better adaptation is very sparse. One of the few studies which apparently supports this view was reported by Slavin et al (1982). These authors assessed over 100 long-term survivors of childhood cancer, and reported that good adjustment was found where the child was informed on diagnosis, or for younger children, by six years of age. Claflin \& Barbarin (1991) reported that parents were less likely to involve younger children (under nine years of age) in discussions about the illness compared with adolescents. The degree of distress reported by the children, however, did not differ. As a consequence, Claflin \& Barbarin (1991) argue that 'telling less' is no more protective, and suggest that there are advantages in involving children directly.

In these situations, it is very difficult to determine what parents mean when they say that they have discussed the illness with the child. This can often mean open discussion about the immediate treatment, but careful avoidance of any discussion of the potentially life-threatening nature of the disease. Neither is it clear what children mean when they say they understand everything. In a recent study involving adolescents treated for bone tumours (Eiser et al, 1997, in press), we attempted to probe for more information about what children meant when they said they knew everything. For many, knowledge meant understanding the orthopaedic problems associated with treatment, to a much greater extent than the implications of chemotherapy. Children were divided into two groups on the basis of their accounts of the diagnostic period; those who remembered being told about the illness on diagnosis $(n=15)$, and those who believed they were not given such information until later $(n=21)$. We were unable to replicate the findings quoted above, finding no association between the timing of information and later adjustment. However, most patients were satisfied with the way in which they had been treated. Those who reported being informed earlier attributed their subsequent adjustment and satisfaction to the fact that they knew what to expect and consequently were better able to cope. Those who were not informed but were nevertheless satisfied argued that they did not need to know about the disease, since they trusted their parents and doctors to do whatever was best for them.

It is unlikely that there will be a simple answer to the question of what children with a lifethreatening disease should be told. The medical treatment itself is complex and we cannot expect all patients (or their parents) to be able to assimilate the details of treatment. More than this, it is important to recognise different ways of coping with threatening information. This recognition seems currently much more embedded in adult health psychology compared with work involving children. Identification of 'monitors' (who seek out information) and 'blunters' who suppress threatening information (Miller, 1995) has some potential in explaining adult responses to chronic disease. Recognition of similar differences in children is necessary in order to avoid the dangers of a prescriptive approach in which it is assumed that information is necessarily a good thing, regardless of the specific situation or characteristics of the individual concerned. Such a distinction is not only likely to be of relevance on diagnosis, but may continue to be important as the child grows up and encounters new situations in which the limitations of the illness become more apparent.

It is frequently asserted that information given to sick children, about their diagnosis, the treatment or the prognosis, must be appropriate for their developmental level (Eiser, 1989). Undoubtedly, many clinicians become expert in tailoring information to the appropriate level. Theoretically, however, we remain somewhat entrenched within stage models, that is children at defined stages are able to understand certain types of information and not others. There are so many exceptions to these approaches that the whole area seems to have stagnated. The question - how to explain chronic illness to a child - remains very real. We must hope that new theoretical approaches emerge in the near future.

\section{Self-care and compliance}

Many parents report difficulties in encouraging their children to accept treatment. The goal of treatment of chronic disease is often seen to involve encouraging the child to become responsible for self-care, and therefore be as independent as possible; this is most clearly seen in conditions such as asthma or diabetes. However, the transfer of responsibility from parent to adolescent can be tricky, with many families experiencing difficulties during the hand-over phase - an important part of good clinical management must involve monitoring this transfer of responsibility.

Unsurprisingly, home-based care is potentially an enormous strain on family life. Yet compliance is important not only from the point of view of the child's health. It is important that patients do as 
they are prescribed so that treatments can be evaluated for their efficacy. Failure to take medication as prescribed can lead to erroneous conclusions about the value of a particular treatment.

It is often assumed that younger children are more compliant with medical advice than adolescents are. In many circumstances, especially in acute illness, this is undoubtedly true. Noncompliance is unfortunately very common, especially among young people with conditions such as asthma or diabetes. La Greca (1990) has noted that non-compliance is most acute in conditions involving complex regimens and those in which treatments are associated with changes in physical appearance.

These differences in compliance have not been noted where the condition is more serious. Phipps \& DeCuir-Whalley (1990) reported that adolescents receiving bone-marrow transplants were more compliant than children undergoing the same procedures. The implication is that adolescents who understand the seriousness of the situation are often very compliant patients. Most importantly, we should not allow our prejudices (that adolescents are rebellious and difficult) to colour our approach to patients.

\section{School liaison}

Children and adolescents with chronic illness live in two worlds. The world of the hospital and treatment may take up a proportion of their time, but they will also be encouraged to participate fully in school life. 'Good' treatment is not just a matter of controlling the disease, but also involves ensuring that the child is educated and prepared for independent adult living. The importance of school, both for the experience of normal social relationships and for the attainment of academic success, has implications for long-term functioning.

The problems children may experience on return to school following diagnosis of a chronic condition have been well described. Children may look different physically, they may experience learning difficulties as a direct result of the condition (e.g. where there is CNS involvement) or simply as a result of extended absences, and they may have difficulties socially in mixing with peers. In a few cases, children may respond by refusing to go to school at all. They are at risk from being bullied or teased, especially when they look small, vulnerable or just different from others. There is considerable scope, therefore, for preventive programmes designed to ease re-integration into the school setting.

Varni et al (1993) report some success using a social skills training programme with children newly diagnosed with cancer. This included assertiveness training, problem-solving and discussion of ways in which to handle problem situations such as teasing. Children who completed the social skills training showed better adjustment at nine-month follow-up, fewer behaviour problems at home and school, and higher selfesteem compared with those who experienced a standard school re-integration programme. (These results seem particularly impressive given that the standard programme involved education and support as well as school conferences and classroom presentations; perhaps much more than is on offer as standard in the UK.)

Although there are advantages in encouraging children to participate fully in school life, we should not forget that they have unique needs. They also face considerable prejudice after school, especially in obtaining work, insurance or mortgages. Perhaps they need to be prepared about this. At the least, they may need more sensitive careers guidance than is routinely available.

\section{Family and ethnic issues}

The way in which a child responds to a chronic condition is closely dependent on the reactions of the family. In diabetes, good haemoglobin control is associated with good family functioning, that is children tend to have better diabetes control when the family functions well together, compared with children from families characterised by greater conflict. Characteristics of good family functioning may change, however, depending on the age of the child. The most successful families are those who recognise the child's needs for independence and are able to support the child through the process of attaining complete responsibility for their own care. Even so, other factors are clearly involved; it is not always the case that families who function well together have children who are in good control. There is some evidence that hormonal changes during adolescence very much challenge the attainment of good control, independently of social or family functioning (Johnson et al, 1992). Good family functioning is helpful, but is not the whole story. Existing research suggests that children from single parent families are more vulnerable than those from intact families, but again the evidence to date is far from conclusive 
(Christiaanse et al, 1989). Given the changing nature and organisation of families, it is imperative that more attention is paid to the special challenges that face children from single parent families, or those who are adopted or fostered. Limited financial resources are undoubtedly an issue. Thought also needs to be given to doctor-family communication. While many paediatricians are careful to include both parents in discussions about the diagnosis and implications of treatment, they can be less responsive to the special needs of non-conventional families. Single mothers often voice their dissatisfaction with diagnostic interviews, where they were given bad news alone and were not given the opportunity to include a friend in the discussion.

We know little about the responses of families from different social and ethnic backgrounds to chronic disease in their child. Major differences must be expected, however, given ethnic differences in understanding and attitude toward disease. There are some exceptions. Some work has been conducted with children suffering from conditions which differentially affect some ethnic groups, such as sickle cell disease or Tay-Sachs disease. In these instances, much greater care needs to be taken over distinguishing between the effects of the disease and those of social class or economic status. It may also be important to develop new instruments for work with these children. The assumption that standardised measures developed for work with White children will be appropriate has little substance.

\section{When a sibling has a chronic illness}

It is widely acknowledged that healthy siblings are especially vulnerable when a child has a chronic illness. They may be separated from the rest of the family, they generally have inadequate explanations about the illness, and their daily routines are markedly interrupted. Younger children can need reassurances that they are not responsible for the illness. Increasingly, there is a recognition that some attempt should be made to involve well siblings in information exchanges or in caring for the sick child.

Again, the earliest ideas, that well siblings will show problems or deviant behaviour, have only partially been supported in the literature. Well siblings have opportunities to develop empathy that are just not available to children in healthy families. Some well siblings certainly do adopt very altruistic behaviours, and subsequently may choose work in the helping professions.

Recognition of their potentially vulnerable status is important, however. Groups for siblings, which encourage sharing of concerns, as well as allowing the sibling to function independently of the nuclear family, appear successful for those who choose to participate. Meyer \& Vadasy (1994) provide a very practical account of running groups for siblings with the aims of sharing frustrations and suggesting ways of coping (see Box 2).

\section{When a parent has a chronic illness}

The diagnosis of a chronic or life-threatening condition in a parent has, of course, major implications for the health and well-being of children in the family. A small amount of empirical work supports the view that children in such families show elevated levels of anxiety and depression around the diagnosis and during the terminal phase of the illness. The extent to which these symptoms are reported, however, is dependent on many factors, but especially the age and gender of the child, as well as gender of the affected parent. Adolescents are more affected than younger children, although greatest distress is reported by adolescent girls whose mother is ill. This may be partly because girls tend to ruminate over problems more than boys, partly because women may use their daughters as confidantes, thereby adding to the girls' involvement and distress. Grant \& Compas (1995) suggest that the problem is most often the result of the greater caretaking burden taken on by adolescent girls compared with boys or younger children. It may also reflect a very real vulnerability and fear experienced by adolescent girls. Much of the research has included daughters of women with breast cancer, and many young girls may experience

Box 2. The best ways to provide information for siblings (from Meyer \& Vadasy, 1994)

Keep the illness an open topic

Answer questions

Provide written materials

Include siblings in hospital visits 
heightened distress, given that some breast cancers are now known to include a genetic component. It is important that future work includes a wider range of diagnoses, in order to tease out the processes underlying the apparent gender differences in how young people cope with parental illness.

Work such as this highlights the practical and emotional difficulties experienced by children, as well as suggesting the heightened vulnerability of some groups. The finding that adolescent girls report more stress than other groups is in line with other work suggesting that women are more vulnerable to health-related stresses. However, it also needs to be considered in relation to the fact that adolescent boys were significantly less likely to agree to participate in the study anyway. It would indeed be a mistake to assume that boys were less distressed than girls, although their withdrawal from the situation may make it more difficult to provide the appropriate help.

\section{Conclusions}

As has been noted previously (Cottrell \& Worrall, 1995), the burden of disclosing the diagnosis of a chronic disease tends to be shouldered by paediatricians. It is also true that paediatricians are often comfortable in dealing with many of the more routine behavioural and emotional problems that can occur among these children or their families. Given the practical demands of treatments, and the emotional consequences of a potentially life-threatening disease for the family, it is impressive how resilient those involved can be. It is appropriate, therefore, that psychiatric referral may be the exception rather than the norm.

The disadvantages of such a model of care are that there may be a reluctance to refer children to a psychiatrist in any but last resort situations. For families, there may well be a stigma associated with such a referral. Other workers, particularly psychologists, social workers or specialist nurses, like to see their role as involving psychological support for families. They may well count such activities high in their job satisfaction. The result, however, may be that referral to psychiatric services is limited to children with major and deepseated problems. Failure to deal effectively with these problems may be seen to be a disincentive to future referrals. Much remains to be done at the level of negotiating professional roles in care of these children, with clearer guidelines needed for paediatricians so that they are better able to judge the most appropriate source of referral for any child.

\section{Acknowledgements}

The Child and Family Research Group is funded by the Cancer Research Campaign, London (CP1019/0101).

\section{References}

Claflin, C. J. \& Barbarin, O. A. (1991) Does telling less protect more? Relationships among age, information disclosure, and what children with cancer see and feel. Journal of Pediatric Psychology, 16, 169-174.

Christiaanse, M. E., Lavigne, J. V. \& Lerner, C. V. (1989) Psychosocial aspects of compliance in children and adolescents with asthma. Developmental and Behavioral Pediatrics, $10,75-80$.

Cottrell, D. \& Worrall, A. (1995) Liaison child and adolescent psychiatry. Advances in Psychiatric Treatment, 1, 78-85.

Drotar, D. (1978) Psychological research in paediatric settings: lessons from the field. Journal of Pediatric Psychology, 19, 63-79.

Eiser, C. (1989) Children's understanding of illness: A critique of the 'stage' approach. Psychology and Health, 3, 93-101.

— \& Jenney, M. E. M. (1996) Measuring symptomatic benefit and quality of life in paediatric oncology. British Journal of Cancer, 73, 1313-1316.

-, Cool, P. \& Grimer, R. (1997) What do children with a lifethreatening illness want to know? Sarcoma, in press.

Grant, K. E. \& Compas, B. E. (1995) Stress and anxiousdepressed symptoms among adolescents: Searching for mechanisms of risk. Journal of Consulting and Clinical Psychology, 63, 1015-1021.

Gortmaker, S. \& Sappenfield, W. (1984) Chronic disorders: Prevalence and impact. Pediatric Clinics of North America, 31, 3-18.

La Greca, A. M. (1990) Social consequences of pediatric conditions: Fertile area for future investigation and intervention. Journal of Pediatric Psychology, 15, 285-307.

Johnson, S. B., Freund, A., Silverstein, J. H., et al (1992) Adherence/health status relationships in childhood diabetes. Health Psychology, 9, 606-631.

Lavigne, J. V. \& Faier-Routman, J. (1992) Psychological adjustment to pediatric physical disorders: A meta-analytic review. Journal of Pediatric Psychology, 17, 133-157.

Meyer, D. J. \& Vadasy, P. F. (1994) Sibshops: Workshops for Siblings of Children with Special Needs. Baltimore, MD: Brookes.

Miller, S. M. (1995) Monitoring versus blunting styles of coping with cancer influence the information patients want and need about their disease. Cancer, 76, 167-177.

Phipps, S. \& DeCuir-Whalley, S. (1990) Adherence issues in pediatric bone-marrow transplantation. Journal of Pediatric Psychology, 15, 385-400.

Slavin, L. A., O'Malley, J. E., Koocher, G., et al (1982) Communication of the cancer diagnosis to pediatric patients: Impact on long-term adjustment. American Journal of Psychiatry, 139, 179-183.

Stein, R. E. K, Bauman, L. J, Westbrook, L. E, et al (1993) Framework for identifying children who have chronic conditions: The case for a new definition. Journal of Pediatrics, 122, 342-347.

Varni, J. W., Katz, E. R., Colegrove, R., et al (1993) The impact of social skills training on the adjustment of children with newly diagnosed cancer. Journal of Pediatric Psychology, 18, 751-768. 
Wallander, J. L. \& Thompson, R. J. (1995) Psychosocial adjustment of children with chronic physical conditions. In Handbook of Pediatric Psychology, vol. 2 (ed. M. C. Roberts), pp. 124-141. New York: Guilford.

\section{Multiple choice questions}

1. Children's adjustment to having a chronic disease:

$a$ is better if they understand exactly what the treatment will be

b varies between individuals

c is better if they are told as soon as possible after diagnosis.

2. Adolescents are more compliant with treatment compared with children if:
a the disease is life-threatening
b treatment affects physical appearance
c treatment regimens are complex.

3. When a parent has chronic disease:
a girls are more distressed than boys
b children are more distressed than adolescents
c children's distress varies with the gender of the parent.

4. When a sibling has chronic disease, it is important to:
a protect other children from the information
b allow them to visit the sick child
c provide written information.

5. Difficulties experienced on return to school can be alleviated by:
a providing other children with information
b assertiveness training for the sick child
c there is little that can be done.

\begin{tabular}{|c|c|c|c|c|}
\hline \multicolumn{5}{|c|}{ MCQ answers } \\
\hline 1 & 2 & 3 & 4 & 5 \\
\hline a $F$ & a $\mathrm{T}$ & a $T$ & a $F$ & a $T$ \\
\hline b $\mathrm{T}$ & b $\mathrm{T}$ & b F & b $\mathrm{T}$ & b $\mathrm{T}$ \\
\hline c $\mathrm{T}$ & c $\mathrm{T}$ & c $\mathrm{T}$ & c $\mathrm{T}$ & c $\mathrm{F}$ \\
\hline
\end{tabular}

